\title{
The Thinking about the Application of Advanced Simulation Equipment in the Clinical Medical Practice Teaching
}

\author{
Wang Chenggang \\ School of Psychology \\ HeBei United University,HEUU \\ Tang Shan,China \\ e-mail:ncmcwcg@163.com
}

\author{
Li Lina \\ School of Psychology \\ HeBei United University,HEUU \\ Tang Shan,China \\ e-mail: lilina3725582@163.com
}

\begin{abstract}
Currently,with the enhancement of self-protection awareness and the faultiness of the medical System and medical education, the medical education is under the intense impact.It is very difficult that the students in the practice clinical skills on patients. With the renewal of the technology and material, the model of medical education was changed. The students can practice the clinical skills on the advanced simulation equipment. Through the use of advanced simulation equipment, it can avoid the emergence of some legal or ethical issues, and improve the quality of medical students' training.
\end{abstract}

Keywords--simulation equipment; Clinical Medicine; clinical skills

Medicine is a very practical subject, the main characteristics of medical education is to combine the theory and Practice, medical students must study the knowledge of diagnosis and treatment by practice, train clinical thinking, master clinical skills ${ }^{[1]}$. Therefore, the focus on the cultivation of medical students is the cultivation of clinical thinking and clinical skills.With the amplification of the legal system, in order to ensure the patients' safety and rights and interests, the state has made strict provisions in the clinical practice of medical students.Therefore, the teachers are not willing to let the students personally operat because of the fear of liability. At the same time, fewer patients cooperate with the teaching practice, which affects the quality of training for clinical medical students.Anciently, the teaching mode that take the patient as the main objects of practice has a great impact, which has been unable to meet the requirements of medical students. The scale of the hospital and the type of departments made the medical students get a little medical knowledge. In view of the status quo of medical education, the advanced simulation equipment was used in medical education, which solves effectively the problem of medical education in the embarrassing situation, and improves effectively the clinical medical students' theoretical level, clinical skills, team spirit and organization and coordination ability without prejudice to the interests of the patients.Simulated medical teaching is a teaching model that medical students make a diagnosis and adopt corresponding treatment measures according to a variety of physiological parameters and clinical manifestations of the "patients", it is a product of the combination of high-end simulation technology and medical education ${ }^{[2]}$.

\section{I .CHARACTERISTICS AND ADVANTAGES OF MEDICAL SIMULATION TEACHING}

\section{A. Simulation teaching based on high-tech and real training} environment

Medical simulation education is an educational subject, it simulated patients instead of real patients in clinical medicine teaching practice according to the simulation technology ${ }^{[3]}$. In today's science and technology condition, the simulated patients enough to mix the spurious with the genuine which is made of advanced materials and advanced computer technology. The simulated patients and normal people are very similar in appearance, regardless of skin color, texture, flexibility, softness , etc. Some high-end and integrated simulation equipments can simulate all kinds of pathological manifestations by computer control. For example, the medical students can practice really the endotracheal intubation by "the patient's head " ,not only that,"the patient's head " can simulate all kinds of difficult airway management such as lock-jaw , tongue edema, laryngismus, etc, simulation equipment can also simulate all kinds of breath sound and heart murmur. In the practice of emergency medicine, the simulation equipments can give different reactions when the medical students operat the simulated patients, which is like on the true patients. The simulation equipments can help students to establish quickly the concept of the image. At the same time, using the simulation equipments can create a simulated ward, simulated operation room, simulated emergency room, even simulated hospital. The students seem to work in the real hospital.

\section{$B$. The students can choose the cases and the times of learning}

It is difficult for students to meet the low incidence of cases in clinical practice, which caused many difficulties for students in the process of learning. When the students have a low incidence of cases, the most medical students will be at a loss, and even delay the diagnosis and treatments of disease because of the lack of experience. For the low incidence of cases, the teachers can maintain the multiple and real cases, and let the students carry out the repeated training which receives very good results. In the learning process, the students can study selectively a case according to their own interests and realize learning at any time. It solves the practical problem that the cases of hospital is 
insufficient and the time can not be controlled during the internship.

\section{Suitable for different stages of learning of students, can adjust the depth and breadth of knowledge}

The medical students need to understand the depth and breadth of knowledge and the difficulty of the training, which will be different at the different stages of learning of the medical students. With the help of medical simulation equipment, the medical students can perform a variety difficulty of the training according to the different learning objectives. Simulation equipment can meet medical students' learning needs at the different stages and professionals. For the low grade medical students, they can practice the individual skills that are in combination with professional theory, such as history-taking, physical examination,tec,this kind of training can be run through the whole process of medical learning, the students can be repeated practice. The medical students can deepen the theoretical understanding and memory of knowledge after they finish a medical professional courses. Medical students' comprehensive training can be used by simulation equipment and the students can play different roles in different special trainings during the medical practice. The Medical students have an overall understanding of the medical work by setting the simulation ward, simulation outpatient and even simulation hospital,etc, which can not only cultivate the students' clinical skills, but also enhance their self-confidence, so that they can adapt better to the work of the hospital.

\section{Safe training process, no harm to the "patient "}

The training of medical students is implemented in the laboratory, the medical students are safe in the whole process, they are not infected and spreaded many diseases from "the patient ". If the medical student have performed an illegal operation on real patients, they will bring pain to the patients and cause damage to the doctor-patient relationship and bring the psychological barriers to himself. Reducing errors by training will lay a solid foundation for the learning of medical students in the future.

\section{$E$. The operation can be repeated with error correction}

It is impossible and not allowed that the same operation is carried out repetitivly on the " patient ", however, it can be implemented in the simulation system, which is the biggest advantage of the simulation system. The students can practice repeatedly in a simulated patient until he is able to master. The improper operation during the operation will not lead to adverse consequences. The teacher will correct the wrong operation timely when it is appearing, which is conducive to enhance the accuracy and standard operation, and will enhance greatly the medical students' self-confidence.

\section{F. The training process can be controlled, it can fed back timely}

The simulation system can slow, stop or restart operation according to the needs in the pathology, spot treatment and emergency rescue,etc. The teachers and the students can control the simulation system actively. In the process of teaching, the teacher can also stop the operation to correct errors and discuss problems for an operation of students. It has a function of the recording and playback that the real environment has been set by computer in the simulation system. The training process of students can be recorded by a variety of ways, when the training is completed, it can be reviewed and discussed and evaluated and discovered the advantages and faults in time, which is an important feedback for teaching, which is beneficial to the teachers to improve the teaching methods and effect, which helps the students master the clinical skills.

\section{G. Be beneficial to cultivate the spirit of teamwork}

Another prominent characteristic of medical simulation teaching is team training, which is common to carry out the diagnosis and treatments for the " patient ", which can not only help each other and improve together and complete the task of learning very well, but also cultivate students' team spirit and promote the mastery and application of medical knowledge to students, which lay a good foundation for future clinical work.

\section{II .THE THINKING ON PERFECTING THE MEDICAL SIMULATION TEACHING}

The medical simulation teaching reflects the importance of simulation teaching in the future in medical education, because it solves some difficult problems in the medical education to a certain extent. Even so, the medical simulation teaching also need to continue to improve in the process of the training of medical students, so that it can play a better function and contribute to the cultivation of medical talents.

\section{A. Put forward higher requirements for teachers}

The simulated medical teaching is a new and different teaching mode, it has changed the traditional model of " teachers teaching + students listen passively", which has been replaced by a new model of " teachers guideing + students think actively", although the teacher does not explain a lot in the whole learning process, the teacher should change the traditional teaching mode and focus on the full interacting of teachers and students. The teachers should spend more time teaching and set the simulative cases and problems that is provided to discussing of case. Therefore, the teachers should master the application of more comprehensive professional knowledge in the present teaching mode. The medical high-end simulation equipment is based on the advanced computer technology. The teachers should master not only the medical knowledge but also the concrete operation of the computer, such as programming,set specific training course. All of this work will be completed on the computer, the teachers must be used it expertly.

\section{B. Need to strengthen humane quality education of the students}

At present, the medical simulation education has been carried out widely in medical colleges. The clinical skills of 
medical students has been improved greatly, and achieved good effects of teaching because of the upgrading of high-end simulation equipment. But admittedly, although the students' clinical skills have been improved, the humanistic quality of students is on the decline. It is very serious that the students' lack of humanistic care and effective communication with the patients. The object of medical research is the people, Medicine pays attention to direct attention to the survival and the ultimate concern of the people, such as power, personality, dignity, health needs and future destiny. Medicine is a human science in essence, it contains a rich cultural meaning and humanistic nature ${ }^{[4]}$. A little care of doctor can bring mood pleasure for the patient, and it can promote the further communication between doctors and patients, even it can affect curative effect. Therefore, an important part of medical education is the humanistic quality education. The students face to SimMan, computer, software in the process of medical simulation teaching. While the students have to face to a living person and need to communicate with people after they are graduated. Higher medical education should been paid more attention to the humane quality education of the students under the medical simulation teaching deepens unceasingly.

\section{Operation and maintenance cost is high}

A series of studies in the European Society for medical education prove that medical simulation has great potential in medical education. While it exposed the main defects, cost and utility ${ }^{[5]}$. The comprehensive simulation training is based on a certain space, equipment and human. According to the current domestic medical education requirements, the cost of establishment of medical simulation center and the maintenance is high. To the simulate clinical area as an example, it includes a control chamber, a plurality of classroom or operating room and an integrated audio/video system, it also need doctors, technicians and administrative personnel, simulation equipment,etc. If a simulated hospital will be established, the cost of human resource, material resource, equipment maintenance is higher.

\section{Reinforcing teaching material construction}

Both the teachers and students have to adapt to the new model of simulation teaching, which has changed the traditional single teaching model. The teaching form is more flexible. In the process of teaching and learning, there must be a set of feasible teaching materials that can guide the teaching.

\section{E. Simulation training and clinical practice combined closely}

"The simulation" is the core of medical simulation education, it makes the medical students master the knowledge of diagnosis and treatment of a disease in a real simulation environment. Compared with the clinical diagnosis and treatment what the true patients accept, The "simulated patients " cann't communicate with doctors, and tell the painful feelings to the doctor in the course of diagnosis and treatment. Therefore, it is important that the simulation practice teaching combine with clinical practice. The practical clinical work combines with clinical skills which the medical students have to study can improve problem-solving ability of medical students'.

\section{F. Improving the evaluation and examination system}

The medical simulation teaching is implemented by the teacher's guidance and the students' active participation. The quality of the students needs a series of indexes to evaluate. The reform of examination mode can be carried out, which can adopt the flexible and diverse forms to assess the medical students' clinical skills examination. For example, Objective Structured Clinical Examination, writte + interview +operational skills, video playback assessment, mutual evaluation of students, self-evaluation of students, etc. The establishment of evaluation system and the objective evaluation to the teachers and the students in the process of teaching and learning, can provide the basis for further improving the quality of teaching.

\section{G. To improve the teaching method}

The traditional teaching methods about medicine include theory teaching, clinical practice, cases discussion, etc. The medical simulation education is a brand-new teaching mode, which is characterized by the student-centered and teacher- subsidiary. The choice of the teaching methods should be benefit to cultivate the students' independent thinking ability. In the process of simulation teaching, we can use different teaching methods, for example, the teaching method of PBL (problem-based learning), cooperative learning and training in groups, cosplay, the use of simulated patients and standardized patients, etc. According to the reformation of teaching method, we can achieve the goal that improving the quality of teaching and medical personnel, cultivating students' ability of independent thinking, enhancing the enthusiasm of students in active learning.

In short, the medical simulation education is a new educational mode which is currently pursued by international medical education ${ }^{[6]}$, it has been accepted generally in the field of the medical education. The adhibition of advanced simulation equipment in the clinical medical practice teaching broadens greatly the ways of medical education and improves the quality of medical education.

\section{REFERENCES}

[1] Shi Shu yu,Shi Wen Hui,Wang Ying,etc. The Situation and Countermeasures of the Teaching Conflicts in Teaching Hospitals. Modern Hospital Management, 2005,3(3):38-39.

[2] Luo Bing,Song Ting,Zou Jun,etc.The Adhibition of Medical Simulation Teaching Combined with PBL in the Teaching of Physical Diagnosis.China Higher Medical Education.2011,(6):55-56.

[3] Dent JA.Harden RM. A Practical Guide for Medical Teachers. Peking:Peking University Medical Press,2008.217-227.

[4] Li Yong,Chen Junguo. Discussion on Humanistic Medicine and Medical Humanity Education. Modern Medicine \& Health.2006,22(11) : 1755-1756.

[5] Liu Jun,Lin Xun. The Overview and the Enlightenment of the 
Hierarchical Application of Medical Simulation Teaching in Europe. Fudan education BBS.2010,8(5):92-96.

[6] Chen Yuqing,Chang Yajie. Application of Simulation Teaching Combined with the Problem Teaching Model in Clinical Teaching. Higher Education Exploration.2012,8:102-104. 